\title{
Bone marrow-derived clonal mesenchymal stem cells inhibit ovalbumin-induced atopic dermatitis
}

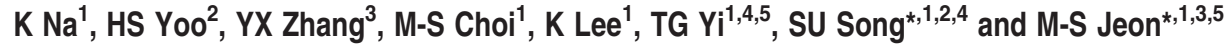

Mesenchymal stem cells (MSCs) possess immunomodulatory activities, including suppression of T- and B-cell activation. However, their effects on atopic dermatitis (AD) have not yet been studied. Using an ovalbumin-induced AD mouse model, we investigated whether MSCs can be used as therapeutics in AD. We isolated both allogeneic and syngeneic clonal MSCs (cMSCs) from mouse bone marrow according to the subfractionation culturing method. Our cMSCs suppressed both T- and B-cell activation. T-cell proliferation and cytokine production, including interferon (IFN)- $\gamma$ and interleukin (IL)-4, were suppressed by inhibition of transcription factors, such as T-bet, GATA-3, and c-Maf. Those transcription factors were nitric oxide dependent. Immunoglobulin E (IgE) suppression occurred through downregulation of AID and BLIMP-1, important regulators for isotype class switch and B-cell differentiation. The cMSCs were injected intravenously into ovalbumin-induced AD mouse model, and the therapeutic effects were analyzed. Injection of both allogeneic and syngeneic cMSCs in an AD mouse model inhibited cell infiltration in skin lesions and decreased the serum level of IgE. IL-4 expression was also suppressed by cMSCs in both the lymph node and skin. The cMSCs migrated to skin lesions and draining lymph nodes. Taken together, these data demonstrated that cMSCs, which suppressed T- and B-cell functions, can be used for the treatment of AD in mice.

Cell Death and Disease (2014) 5, e1345; doi:10.1038/cddis.2014.299; published online 17 July 2014

Atopic dermatitis (AD), also known as atopic eczema, is a very common inflammatory skin disorder and affects $\sim 5-20 \%$ of children worldwide. The incidence of $A D$ has increased over the past 20 years, particularly in Africa, Eastern Asia, Western Europe, and parts of Northern Europe. ${ }^{1}$ The pathogenesis of acute $A D$ is associated with Th2-dominant inflammation, characterized by dermal infiltration of $\mathrm{CD}^{+}{ }^{+} \mathrm{T}$ cells and eosinophils and increased levels of immunoglobulin $\mathrm{E}(\mathrm{IgE})$ and Th2 cytokines. ${ }^{2}$ There is no known cure for AD. There are several treatment approaches for AD such as emollients, topical glucocorticosteroids, calcineurin inhibitors, phototherapies, and immunosuppressants such as cyclosporine $A^{3.4}$ These therapies reduce inflammation, but they also cause side effects. ${ }^{4}$ Therefore, development of new therapeutic approaches is necessary for AD treatment.

Mesenchymal stem cells (MSCs) exert immunosuppressive effects, including suppression of T-cell proliferation, inhibition of dendritic cell function, suppression of B-cell proliferation and terminal differentiation, and immunomodulation of other immune cells such as natural killer (NK) cells and macrophages. ${ }^{5,6}$ Because of their ability to modulate immune responses, MSCs are considered a therapeutic source for the treatment of patients with inflammation-related diseases, such as graft-versus-host disease (GvHD), ${ }^{7}$ collagen-induced arthritis (CIA), ${ }^{8,9}$ experimental autoimmune encephalomyelitis
$(E A E),{ }^{10}$ systemic lupus erythematosus, ${ }^{11}$ sepsis, ${ }^{12}$ acute

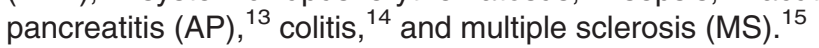

In terms of the immunomodulation mechanism of MSCs, it has been suggested that MSC-mediated immunosuppression requires the preliminary activation of MSCs by immune cells through the secretion of the proinflammatory cytokine interferon (IFN)- $\gamma$ alone or in conjunction with tumor necrosis factor (TNF)- $\alpha$, interleukin (IL)- $1 \alpha$, or IL-1 $\beta .^{16,17}$ An in vivo GvHD model further substantiated such views by demonstrating that IFN- $\gamma$ is necessary for MSCs to suppress disease development. ${ }^{18}$ Therefore, Th1-mediated diseases are mostly selected for treatment by MSCs; however, the effects of MSCs on Th2-related diseases have not been studied extensively. A few recent studies have demonstrated the effects of MSCs on allergic diseases such as rhinitis and asthma. ${ }^{19-22}$ However, the effects of MSCs on AD have not been fully explored. We isolated both allogeneic and syngeneic clonal MSCs (cMSCs) from mouse bone marrow by using the subfractionation culturing method (SCM) and established cMSC lines. ${ }^{23,24}$ We hypothesized that MSCs exert their immunomodulatory effects on allergic inflammation in skin disorders. To test this hypothesis, we administered syngeneic and allogeneic cMSCs into ovalbumin (OVA)-induced $A D$ mice and evaluated their therapeutic effects.

\footnotetext{
${ }^{1}$ Translational Research Center, Inha University School of Medicine, Incheon, Republic of Korea; ${ }^{2}$ Department of Drug Development, Inha University School of Medicine, Incheon, Republic of Korea; ${ }^{3}$ Department of Molecular Biomedicine, Inha University School of Medicine, Incheon, Republic of Korea; ${ }^{4}$ SCM Lifescience Co. Ltd, Incheon, Republic of Korea and ${ }^{5}$ Inha Research Institute for Medical Sciences, Inha University School of Medicine, Incheon, Republic of Korea

*Corresponding authors: SU Song, Translational Research Center, Inha University School of Medicine, 7-206, 3-Ga, Shinheung-Dong, Jung-Gu, Incheon 400-711, Republic of Korea. Tel: +82 32890 2460; Fax: +82 32891 2277; E-mail: sunuksong @inha.ac.kr

or M-S Jeon, Translational Research Center, Inha University School of Medicine, 7-206, 3-Ga, Shinheung-Dong, Jung-Gu, Incheon 400-711, Republic of Korea. Tel: +82 32890 3682; Fax: +82 32890 2462; E-mail: msjeon@inha.ac.kr

Abbreviations: $A D$, atopic dermatitis; MSC, mesenchymal stem cell; cMSC, clonal mesenchymal stem cell; LPS, lipopolysaccharide; SCM, subfractionation culturing method; OVA, ovalbumin; CSR, class switch recombination

Received 02.1.14; revised 20.5.14; accepted 11.6.14; Edited by Y Shi
} 


\section{Results}

Characterization of bone marrow-derived cMSCs. The cMSCs were isolated from the bone marrow of allogeneic $(\mathrm{C} 3 \mathrm{H} / \mathrm{HeN})$ and syngeneic (Balb/c) mice according to our newly established isolation protocol, SCM, as described in the Materials and Methods section. ${ }^{23,24}$ The cell surface epitopes of the cMSCs were analyzed by flow cytometry. The results revealed that the cMSCs were strongly positive for Sca-1, CD44, CD73, and CD105, and were negative for major histocompatibility complex (MHC) class II, CD45, CD103, and CD117. Allogeneic cMSCs were differentiated into osteocytes and chondrocytes, whereas syngeneic cMSCs were differentiated into adipocytes and chondrocytes (Supplementary Figure S1).

The cMSCs suppress T-cell proliferation and cytokine production. Next, we examined T-cell proliferation to test the immunosuppressive potential of cMSCs. Splenocytes were stimulated with anti-CD3 and anti-CD28 antibodies in the presence of CMSCs. T-cell proliferation was measured by thymidine incorporation. As shown in Figure 1, T-cell proliferation was significantly inhibited by both syngeneic and allogeneic cMSCs, even under 1:100 (cMSC/splenocytes) co-culture conditions. IFN- $\gamma$ and IL-4 production were significantly suppressed by both cMSCs, and this suppression was cell-cell contact dependent. When we used a Transwell system, cMSCs did not inhibit cytokine productions (Figure 2a). Cytokine suppression by cMSCs occurred at the level of transcription (Figure 2b). Both T-bet and GATA-3, which are transcription factors for IFN- $\gamma$ and $I L-4$, respectively, were suppressed by cMSCs (Supplementary Figure S2). Inhibition of $\mathrm{T}$-cell proliferation and IFN- $\gamma$ production were rescued in the presence of the nitric oxide (NO) inhibitor, L-NMMA, suggesting that inhibition of T-cell proliferation and IFN- $\gamma$ production were NO dependent (Figure 2c). However, suppression of IL-4 production was not NO dependent. Although the suppression of GATA-3 and c-Maf, which are Th2-type transcription factors, by cMSCs was rescued by the NO inhibitor, IL-4 expression was still inhibited by cMSCs (Supplementary Figure S2). The cMSCs differentially regulate IL-4 cytokine production compared with IFN- $\gamma$ expression.

The cMSCs suppress B-cell proliferation and IgE production. A few recent studies have elucidated the effects of MSCs on B cells, including inhibition of terminal differentiation. ${ }^{25}$ However, the regulation of IgE by MSCs has not yet been reported. Therefore, we investigated whether cMSCs directly inhibited IgE production of B cells. For the
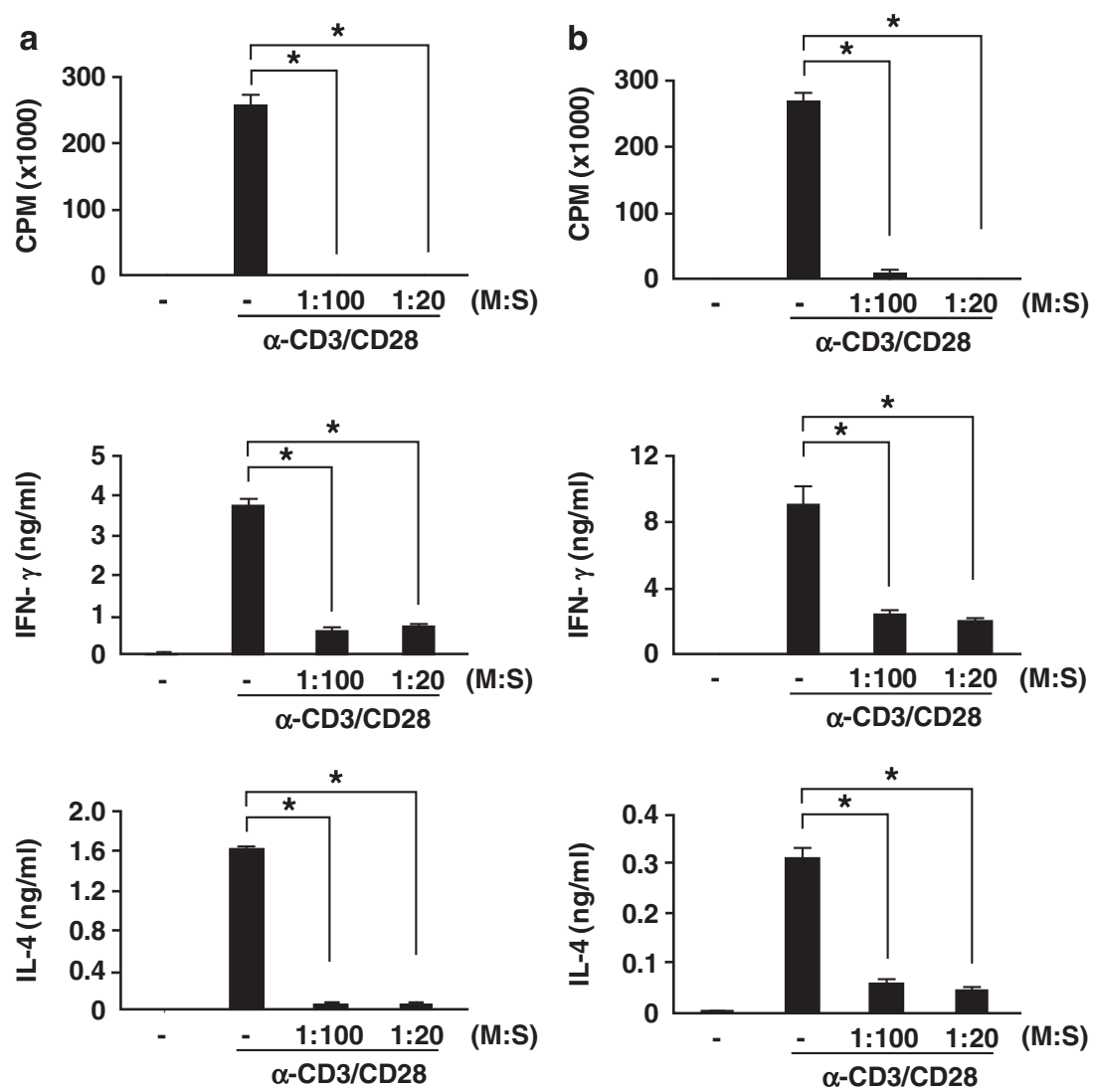

Figure 1 Suppression of T-cell proliferation and cytokine production by cMSCs. Splenocytes from Balb/c mice were stimulated with anti-CD3 and anti-CD28 antibodies in the presence or absence of cMSCs. Cells were cultured for 3 days. During the final $16 \mathrm{~h}$ culture period, $1 \mu \mathrm{Ci}{ }^{3} \mathrm{H}$-thymidine was added and T-cell proliferation was determined by thymidine incorporation. Both syngeneic (a) and allogeneic (b) cMSCs significantly inhibited T-cell proliferation in a number-dependent manner. After $48 \mathrm{~h}$ of stimulation, IFN- $\gamma$ and IL-4 levels were measured by ELISA. Both cMSCs inhibited IFN- $\gamma$ and IL-4 production. Similar results were obtained in three independent experiments. M, cMSCs; S, splenocytes, ${ }^{*} P<0.001$ 

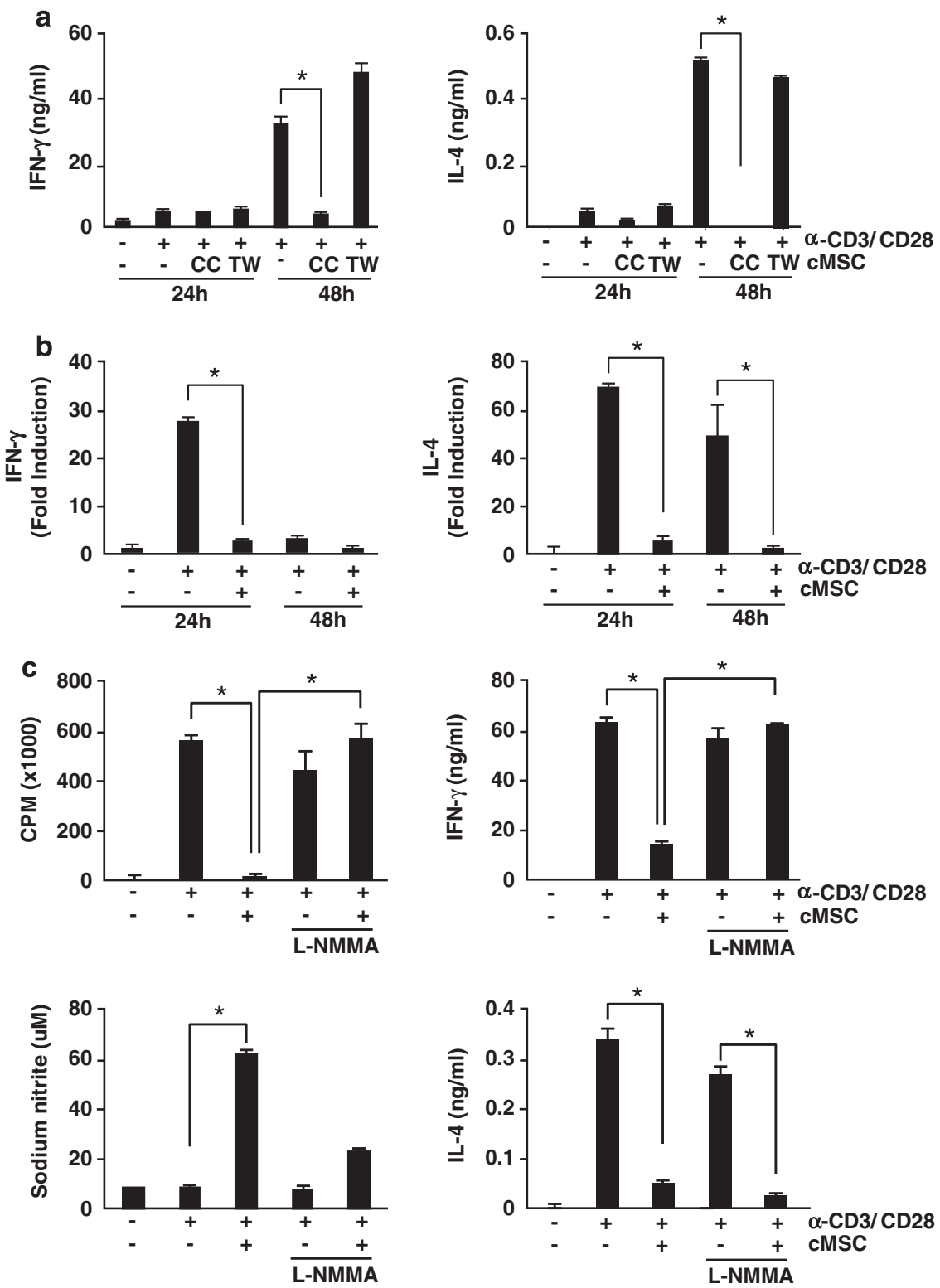

Figure 2 Cell-cell contact- and NO-mediated T-cell suppression by cMSCs. Splenocytes from Balb/c mice were stimulated with anti-CD3 and anti-CD28 antibodies in the presence of allogeneic cMSCs. The 24-well Transwell plates with a $0.4-\mu \mathrm{m}$ pore membrane were used to separate the splenocytes from the cMSCs. After $48 \mathrm{~h}$ of stimulation, IFN- $\gamma$ and IL-4 protein levels were measured by ELISA. (a) The cMSCs inhibited IFN- $\gamma$ and IL-4 production in a cell-cell contact-dependent manner. (b) IFN- $\gamma$ and IL- 4 mRNAs were also inhibited by cMSCs. (c) To prove NO involvement, $1 \mathrm{M} \mathrm{L-NMMA}$ was added in culture media. The NO inhibitor L-NMMA rescued cMSC-mediated T-cell proliferation and IFN- $\gamma$ production but not IL-4 production. Similar results were obtained in three independent experiments. CC, co-culture; TW, Transwell, ${ }^{*} P<0.001$

induction of IgE in vitro, purified B cells were stimulated with lipopolysaccharide (LPS) ${ }^{26}$ or anti-CD40 antibodies in the presence of $\mathrm{IL}-4 .{ }^{27,28}$ Under these conditions, we first investigated whether allogeneic cMSCs affect B-cell proliferation. The cMSCs were co-cultured with B cells and proliferation and cell division were measured. Although cMSCs inhibited B-cell proliferation under both conditions, cell division was more significantly blocked by cMSCs in the presence of anti-CD40 antibody than LPS (Figure 3a and Supplementary Figure S3). IgE production in culture media was significantly suppressed by cMSCs, and this suppression was cell-cell contact dependent (Figure $3 b$ ). We also measured the number of IgE-producing cells by flow cytometry. IgE-producing cells were significantly suppressed by cMSCs (Figure 3c). Similar results were obtained in another experiment with syngeneic cMSCs (Supplementary Figure S4). These results suggested that cMSCs inhibit not only B-cell proliferation but also IgE production via cell-cell contact.

Inhibition of class switch DNA recombination and plasma cell differentiation by cMSCs. Germline transcription from isotype-specific promoters is required for class switch DNA recombination (CSR). ${ }^{28}$ We investigated whether IgE suppression by cMSCs reflects the inhibition of CSR. The levels of germline transcripts (GLTs) and 

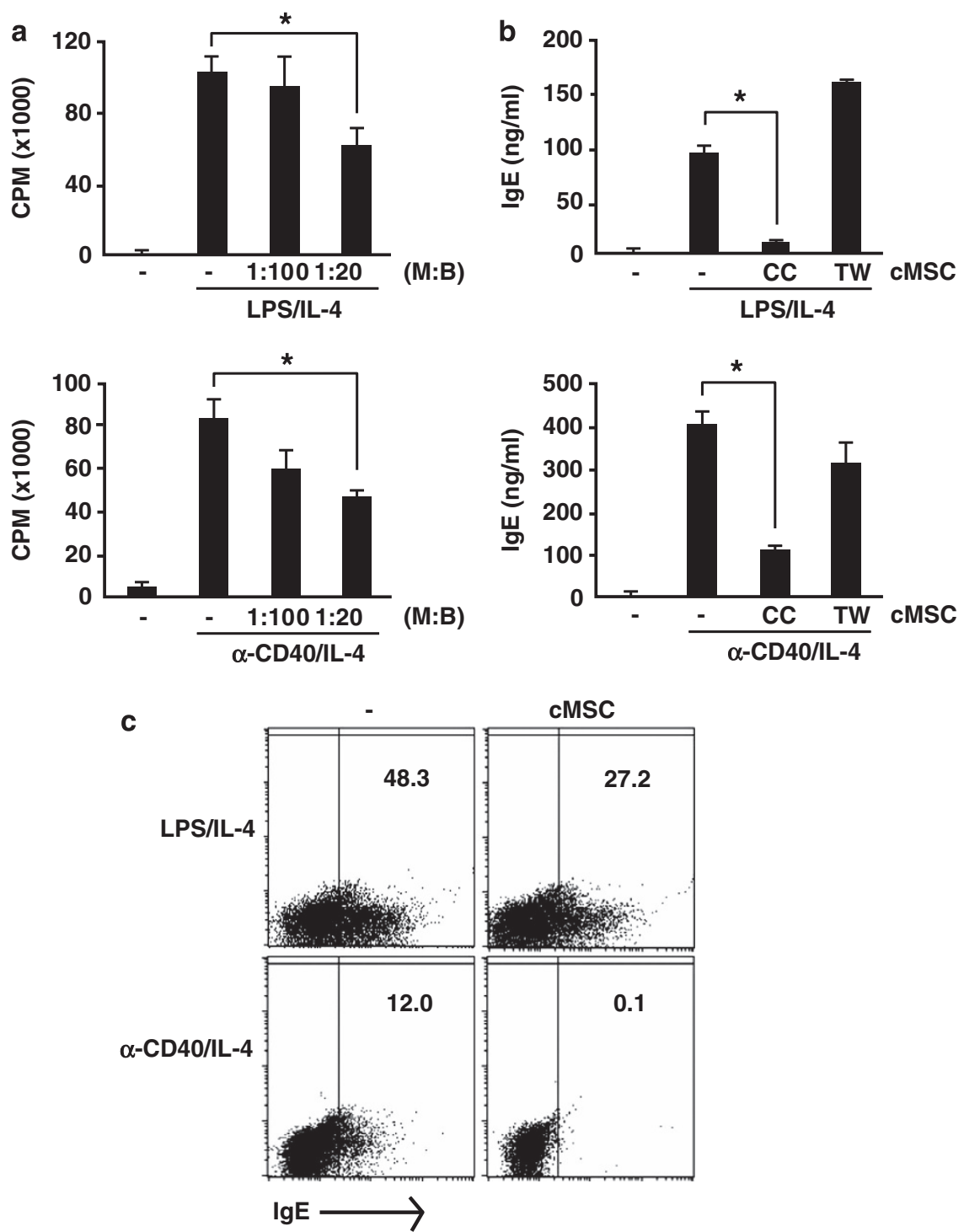

Figure 3 Suppression of B-cell proliferation and IgE production by cMSCs. (a) Purified B cells from Balb/c mice were stimulated with either LPS or anti-CD40 antibodies in the presence of IL-4. Cells were cultured for 3 days. During the final $16 \mathrm{~h}$ culture period, $1 \mu \mathrm{Ci}{ }^{3} \mathrm{H}$-thymidine was added and B-cell proliferation was determined by thymidine incorporation. The cMSCs slightly inhibited B-cell proliferation in a number-dependent manner. (b) To measure lgE production, cells were cultured for 5 days and lgE in culture media was measured by ELISA. IgE production was inhibited by cMSCs in a cell-cell contact-dependent manner. (c) To measure IgE-producing B cells, cells were cultured for 3 days. During the final $6 \mathrm{~h}$ culture period, brefeldin A was added. Intracellular IgE production was detected by flow cytometry. IgE-producing B cells were reduced by cMSCs. Similar results were obtained in two to three independent experiments. M, cMSCs; B, B cells; CC, co-culture; TW, Transwell, ${ }^{*} P<0.001$

post-switch transcripts (PSTs) were examined by reverse transcription-PCR (RT-PCR) on days 2 and 3. $\varepsilon$-GLT and $\varepsilon^{-}$ PST are regarded as the hallmarks of IgE isotype class switching. ${ }^{29}$ As shown in Figure $4 a$, the levels of both $\varepsilon$-GLT and $\varepsilon$-PST were significantly decreased by $\mathrm{CMSC}$ s under LPS/IL-4 stimulated conditions, whereas both transcripts were weakly decreased by cMSCs under anti-CD40/IL-4 stimulation. These results indicate that cMSCs suppress IgE production via different mechanisms, according to stimulation.

Several factors are important for CSR and plasma cell differentiation, such as BCL-6, PAX5, activation-induced cytidine deaminase (AID), XBP1, and BLIMP-1. ${ }^{30}$ Therefore, we measured their mRNA expression by real-time RT-PCR. Under stimulation, expression levels of $B C L-6$ and $P A X 5$ were decreased, whereas expression levels of $X B P-1, A I D$, and $B L I M P-1$ were increased, indicating that $B$ cells under both conditions were in the process of CSR and differentiation into plasma cells. However, expression of PAX5 and XBP-1 did not change by CMSCs, whereas the expression of BLIMP-1 was significantly decreased and BCL6 expression was slightly increased in the presence of CMSCs. Intriguingly, both protein and mRNA expression of Blimp-1 were significantly decreased by cMSCs, whereas the mRNA level of AID was increased but the protein level was significantly decreased by cMSCs (Figures $4 \mathrm{~b}$ and $\mathrm{c}$ ). These findings suggest that Blimp1 and AID may be transcriptionally and post-transcriptionally regulated by CMSCs, respectively. Collectively, these results indicate that CMSCs affect both CSR and B-cell differentiation by inhibiting $A I D$ and BLIMP-1. 

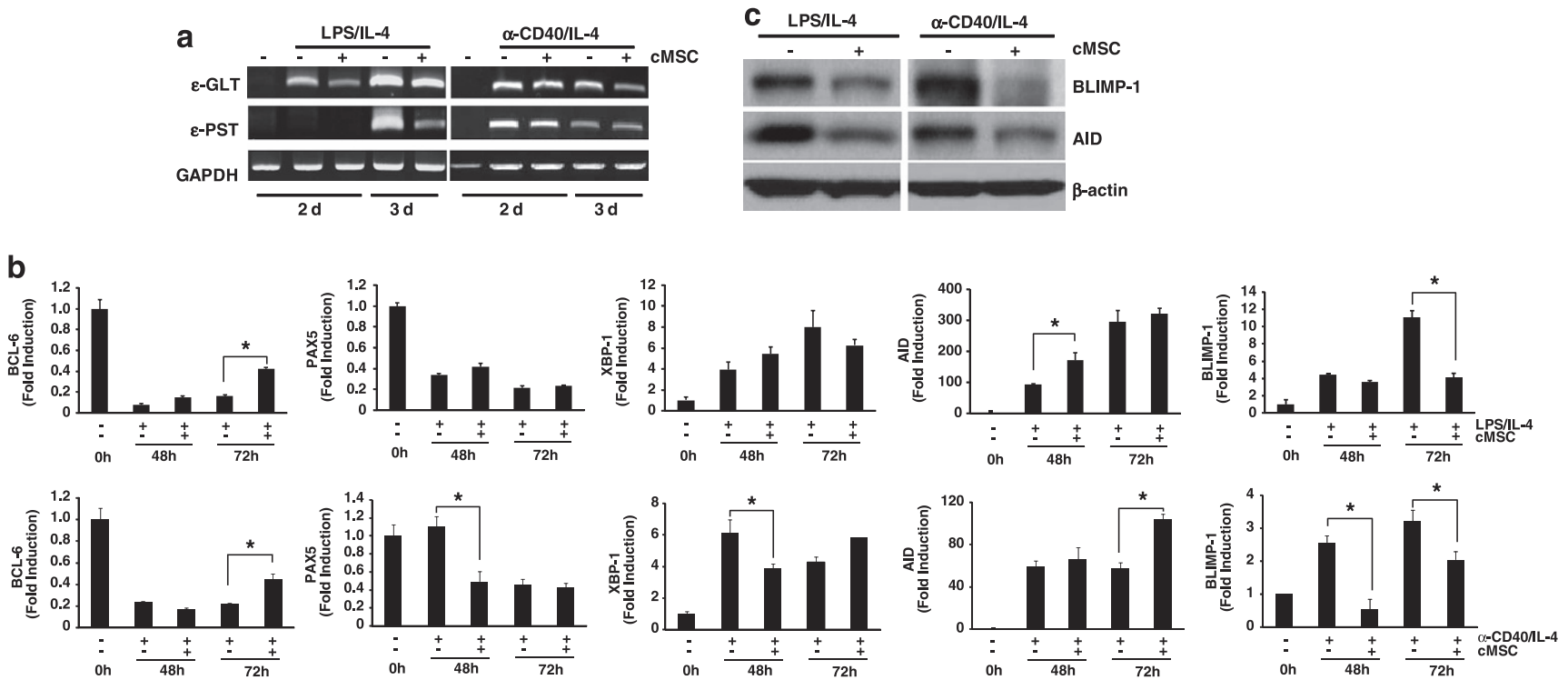

Figure 4 Suppression of isotype class switching and B-cell differentiation by cMSCs. (a) RNA was isolated from stimulated B cells. $\varepsilon$-GLT and $\varepsilon$-PST mRNA levels were significantly reduced in LPS/IL-4-stimulated B cells by CMSCs, whereas both transcripts were weakly decreased by cMSCs under anti-CD40//L-4 stimulation. (b) Other transcription factors for B-cell differentiation were analyzed by real-time PCR. (c) The cMSCs inhibited BLIMP-1 and AID protein expression in B cells cultured for 4 days. Similar results were obtained in two to three independent experiments. ${ }^{*} P<0.001$

The cMSCs suppress AD in the mouse model. It is now generally accepted that MSCs are hypoimmunogenic as they do not express MHC class II. ${ }^{31}$ However, we wanted to exclude the allograft rejection response in this $A D$ mouse model. Therefore, we used both allogeneic and syngeneic cMSCs. Using cMSCs that suppressed both $\mathrm{T}$ cells and B cells, we examined the therapeutic effects of both allogeneic and syngeneic cMSCs on AD. We used the OVA-induced AD mouse model. ${ }^{32}$ Mice were intraperitoneally sensitized with $10 \mu \mathrm{g}$ of OVA on days 1,7 , and 14 , and epicutaneously sensitized in patches with $10 \mu \mathrm{g}$ of OVA in $100 \mu \mathrm{l}$ of phosphate-buffered saline (PBS). Epicutaneous sensitization comprised two 1-week exposures to OVA. The cMSCs $\left(2 \times 10^{5}\right.$ cells) were intravenously injected 3 times between the epicutaneous sensitization procedures (Figure 5a). Decreased cell infiltration in the skin was observed in mice treated with both allogeneic and syngeneic cMSCs compared with that in the PBS control group (Figure 5b). Severity score of skin lesions and thickness of epidermis and dermis were significantly decreased by cMSCs (Figure $5 c$ ). Mast cells, $\mathrm{T}$ cells, and eosinophils were identified by toluidine blue, anti-CD3 antibodies, and H\&E staining, respectively. These cells were also significantly decreased by cMSCs (Figures $5 \mathrm{~b}$ and $\mathrm{c}$ ). The total and OVA-specific IgE levels were significantly decreased in sera of mice treated with both cMSCs. The total level of IgG2a was significantly increased by both cMSCs, but OVA-specific IgG2a was not affected (Figure 5d). IL-4 expression in lymph nodes and skins were also examined. IL-4 mRNA expression in these tissues was significantly decreased by cMSCs (Figure 5e), consistent with the in vitro result (Figure 1). Expression of IFN- $\gamma$, IL-10, and TGF- $\beta$ did not change by cMSCs in lymph nodes. Expression of Foxp3, which is a marker for regulatory T cells, was also not affected by cMSCs (Figure 5f). These results indicate therapeutic effects on $A D$ by both allogeneic and syngeneic cMSCs.

The cMSCs migrated to skin lesions and draining lymph nodes in the AD mouse model. To examine the cMSC migration pattern in vivo, CM-Dil-stained cMSCs were intravenously injected into PBS control mice and AD mice (Figure 6a). After 3 days, CM-Dil-cMSCs were analyzed by confocal microscopy. The cMSCs were only detected in the epicutaneously sensitized skin of AD mice but not in the skin of PBS-injected control mice, whereas cMSCs in the lung were comparable between PBS and AD mice (Figure 6b). This finding suggests that cMSCs migrate into the area of inflammation. To compare the cMSC migration pattern between lesional and nonlesional skin in $A D$ mice, OVA patches were attached to only the left side of mice (Figure 6a). At 3 days after CM-Dil-stained cMSC injection, axillary lymph nodes were analyzed. More cMSCs were detected in the left lymph node that was located near the lesion (Figure 6c). This result suggests that cMSCs may migrate to the area of inflammation through lymph nodes near the lesions, and then possibly suppress the IL-4 expression by inhibiting effector cells (Figure 5e).

\section{Discussion}

Here, we demonstrated for the first time that cMSCs suppress OVA-induced $A D$ in mice, probably through the inhibition of IL-4 and IgE production. As MSCs have been shown to suppress Th1 cytokine production in $\mathrm{T}$ cells and to require IFN- $\gamma$ to exercise their suppressive capability, MSCs have been frequently used in the treatment of Th1-mediated diseases such as CIA, EAE, and SLE. ${ }^{9-11}$ Consequently, MSCs are less likely to be used in the treatment of 
Th2-mediated diseases. Several recent studies have shown that MSCs can prevent allergic airway inflammation. ${ }^{19-21}$ However, the effects of MSCs on Th2 responses are controversial. Some studies have found that IL-4 production is significantly increased in the presence of human MSCs during Th2 differentiation. ${ }^{33-36}$ On the other hand, other studies have found that MSCs reduce IL-4 and IL-5 production. ${ }^{37-39}$ The differences between the studies may have resulted from the use of different MSC lines. MSCs have been well characterized in the treatment of GvHD. However, our recent report showed that different MSC lines had different efficacy on the treatment of GvHD, ${ }^{40}$ suggesting that the immumodulatory properties of each MSC line may vary.

We isolated cMSCs from the bone marrow of Balb/c and $\mathrm{C} 3 \mathrm{H}$ mice. These cMSCs suppressed both Th1 and Th2 responses and even $\lg \mathrm{E}$ production of $\mathrm{B}$ cells. Using a Transwell system, we demonstrated that the inhibition of IFN- $\gamma$ and IL-4 production by cMSCs was cell-cell contact dependent. The molecular mechanism and the identity of the cell surface molecule(s) responsible for the inhibition are still unknown. However, both cytokines appear to be transcriptionally regulated because $T$-bet, $c-M a f$, and GATA-3

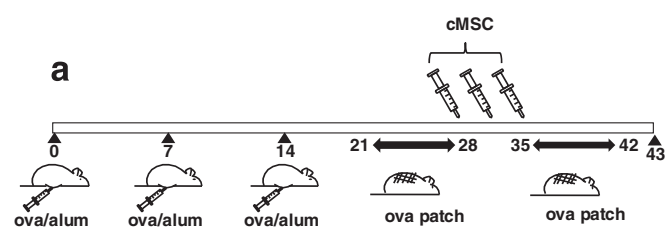

b

OVA patch

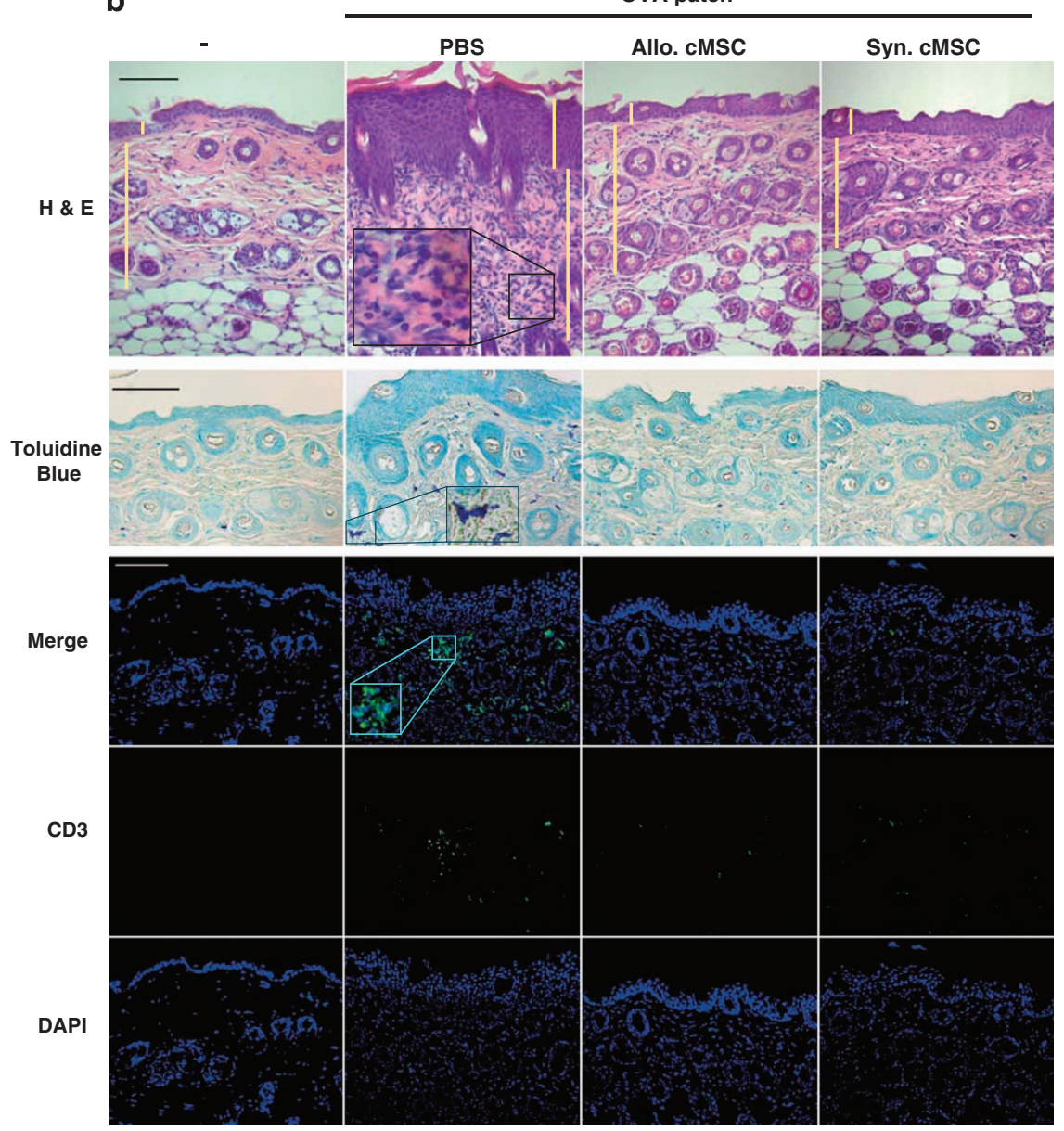

Figure 5 Suppression of AD by cMSCs. (a) Scheme of AD mouse model. Mice were immunized with OVA/Alum on days 0,7 , and 14 and epicutaneously sensitized with OVA patches on day 21 for 1 week. The cMSCs were intravenously injected 3 times between the epicutaneous sensitization procedures. The mice were killed on day 43, and the harvested OVA patch skin lesions. (b) Hematoxylin and eosin (H\&E) staining of mice treated with PBS or cMSCs. Toluidine blue staining (lower panels) shows mast cell infiltration in mice skin. T cells in skin specimens were identified by FITC-conjugated anti-CD3 antibodies. Data are representative of three experiments. (c) Skin scoring, thickness, and leukocyte counts in mice skin shown as mean \pm S.E.M.; $n=6$ mice per group. HPF, high-powered field $(\times 400)$. (d) The serum levels of total and OVA-specific antibodies were measured by ELISA. (e) IL-4 mRNA was detected either in lymph nodes or skin lesion, as determined by real-time PCR. (f) Levels of several cytokines in draining lymph nodes were determined by real-time PCR. (d-f) Each single dot in the graphs shows individual mouse results. Similar results were obtained in two to three independent experiments. Allo, allogeneic; Syn, syngeneic. ${ }^{\dagger} P<0.05,{ }^{*} P<0.01$ and ${ }^{* \star} P<0.001$. Scale bar $=100 \mu \mathrm{m}$ in all photomicrographs 

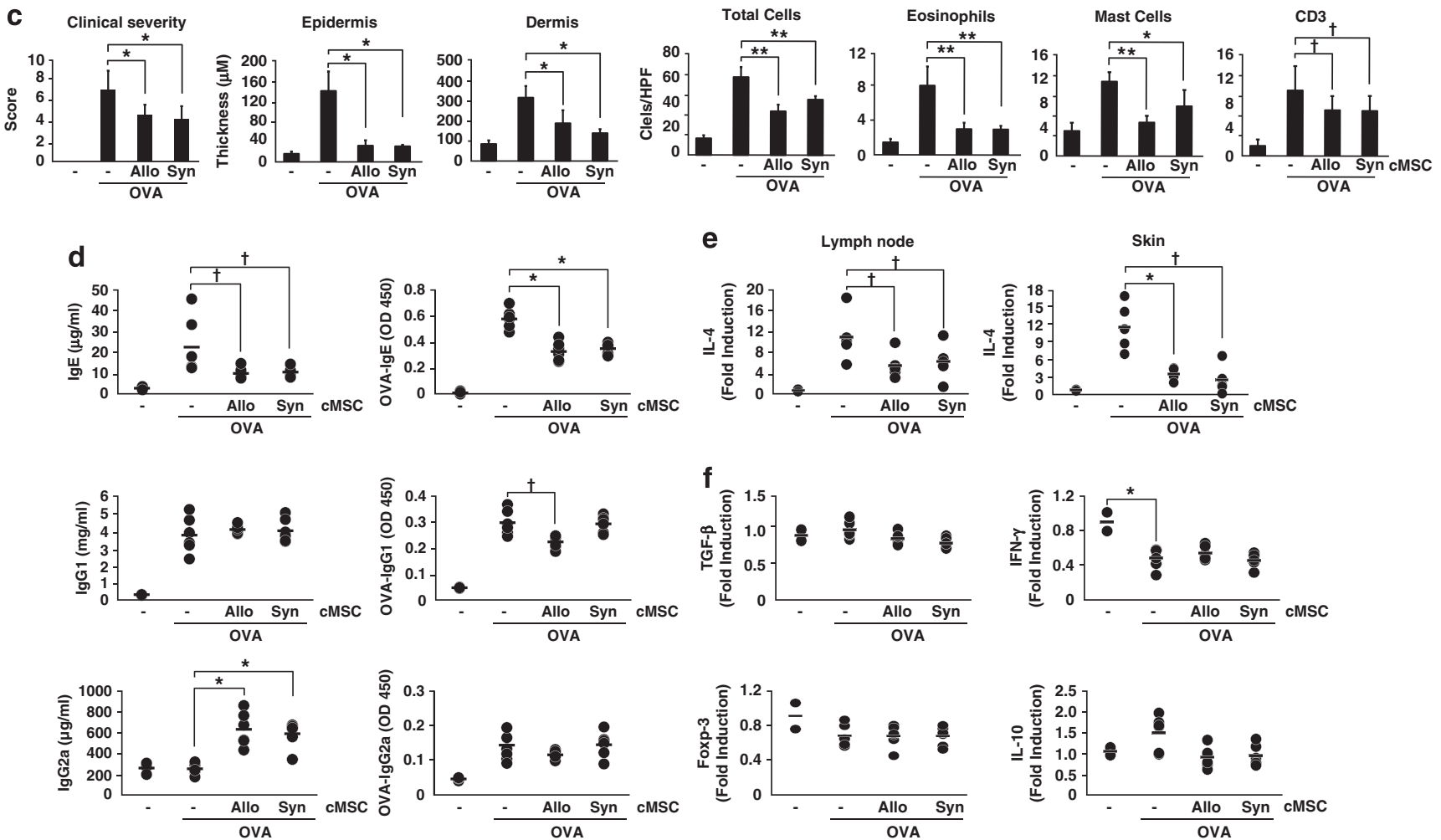

Figure 5 (Continued)

expression levels were decreased by cMSCs. These transcription factors were also NO dependent and were responsible for IFN- $\gamma$ inhibition but not for IL-4 expression by cMSCs (Supplementary Figure S2). The molecular mechanisms by which cMSCs inhibited IL-4 expression should be elucidated in the future.

In addition, we demonstrated for the first time that cMSCs directly inhibited the $\operatorname{lgE}$ production of $\mathrm{B}$ cells that were stimulated with either LPS or anti-CD40 antibodies in the presence of IL-4. We also observed decreased IgG1 expression by cMSCs (Supplementary Figure S4). As our cMSCs express the IL- 4 receptor but not TLR4 on their cell surface, it can be argued that cMSCs can uptake recombinant IL-4 from the medium, resulting in IgE suppression. We excluded this possibility in our study. When we used a Transwell system, cMSCs did not suppress IgE production; this would be the case if the IL-4 receptors expressed on cMSCs interfered with IgE expression. The inhibition of IgE was cell-cell contact dependent. Therefore, it is likely that other factors are involved in suppression of IgE by cMSCs.

The cMSCs significantly inhibited $\varepsilon$-GLT and $\varepsilon$-PST in B cells stimulated with LPS/IL-4. The efficiency of CSR critically depends on the level of AID that is tightly regulated in a B-cell differentiation stage-specific manner. ${ }^{30,41}$ For example, the NF- $k \mathrm{~B}$ p52 subunit and the p65 subunit regulate AID expression by binding to the $A I D$ gene promoter and upstream enhancers, respectively. ${ }^{42,43}$ In this study, AID mRNA did not change by cMSCs, implying that NF- $\kappa$ B activation is not affected by cMSCs. However, the protein level of AID was significantly inhibited by cMSCs. As AID is regulated by
microRNAs, such as miR-155, ${ }^{44,45}$ we investigated whether CMSCs affect miR-155 expression in B cells. We observed that miR-155, which is responsible for AID regulation, was induced in the presence of cMSCs ( $\mathrm{K} \mathrm{Na}$, unpublished data). The cMSCs could negatively regulate AID expression probably via the upregulation of microRNAs such as miR155. In contrast to AID, BLIMP-1 mRNA was significantly inhibited by cMSCs, suggesting that CMSCs may affect transcription factors regulating the BLIMP-1 expression.

When both syngeneic and allogeneic CMSCs were intravenously administered in OVA-induced AD, cell infiltration into the skin lesions and IgE production in sera were significantly decreased compared with those observed in the PBS-treated control mice. OVA-specific IgG1 production was significantly decreased by allogeneic cMSCs but not by syngeneic cMSCs, whereas both cMSCs did not affect OVA-specific IgG2a production. Allogeneic cMSCs appeared to be better for therapeutic effects of the AD than syngeneic cMSCs. However, both cMSCs significantly increased total IgG2a production in sera. It has been reported that MSCs express $\mathrm{IL}-27,{ }^{46}$ which induces IgG2a in B cells. ${ }^{47}$ We speculate that administration of cMSCs may induce IL-27 that results in $\lg$ G2a induction in sera.

There have been several reports showing that MSCs suppress Th2-mediated diseases such as allergic airway inflammation and allergic rhinitis via regulatory T-cell responses. ${ }^{20}$ However, we could not detect a difference in Foxp3 ${ }^{+} \mathrm{CD}^{2} 5^{+} \mathrm{CD}^{+}{ }^{+} \mathrm{T}$ cells after cMSC treatment. In contrast, we could observe a significant decrease in CD25 ${ }^{+}$ $\mathrm{CD}^{+}{ }^{+} \mathrm{T}$ cells in the presence of $\mathrm{cMSC}$ shen we stimulated 

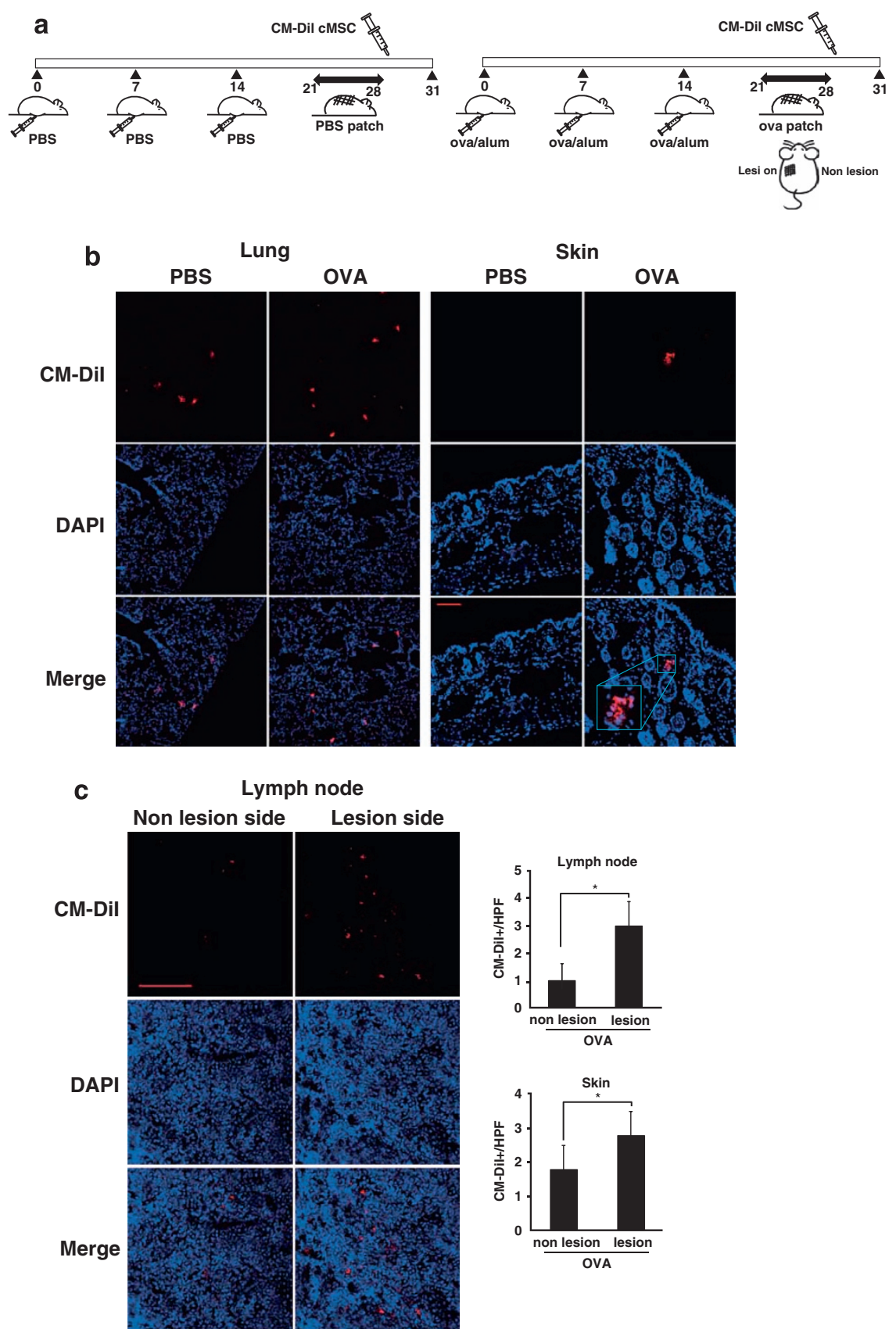

Figure 6 Migration of cMSCs to a skin lesion and draining lymph nodes. (a) Scheme of treatment with CM-Dil-stained cMSCs in PBS control mice and OVA-induced AD mice. Mice were immunized with either PBS or OVA/Alum on days 0,7 , and 14 and epicutaneously sensitized with either PBS or OVA patches on day 21 for 1 week. On day 28, CM-Dil-stained cMSCs were intravenously injected into PBS control mice and AD mice. (b) Mice were killed on day 31, and skin and lungs were harvested. The cMSCs were detected in the lung and OVA-induced skin lesion. (c) OVA patches were treated on only the left side of the skin. At 3 days after CM-Dil-stained cMSCs injection, more cMSCs were detected in the left-side axillary lymph node than the right-side lymph node. CM-Dil-labeled cMSCs were counted in high-powered-field (HPF, $\times 400)$. Similar results were obtained in two independent experiments. ${ }^{*} P<0.001$. Scale bar $=100 \mu \mathrm{m}$ in all photomicrographs

splenocytes with anti-CD3 and anti-CD28 antibodies (data not shown). Furthermore, Foxp3 expression in lymph nodes did not change after administration of cMSCs in AD mice (Figure 5f). These results suggest another mechanism in addition to regulatory $T$ cells.

The cMSCs were observed only in the OVA-induced skin lesion but not in PBS-treated mice, and this suggests that cMSCs can migrate into an inflamed skin area, even by intravenous administration. Intriguingly, more cMSCs were detected in the left-side lymph nodes near the lesion area when we boosted the antigen response on only the left side of the skin area using OVA patch. This suggests that cMSCs may migrate to skin lesions through draining lymph nodes. The reduced cell infiltration in the skin lesion after administration of cMSCs could be because of migration of some cMSCs into the inflamed area and may be resulting in reduced 
generation of effector T cells. The other portion of cMSCs may remain in secondary lymphoid organs to block the migration of effector $\mathrm{T}$ cells to the inflammatory region. More studies should be performed to confirm this speculation.

In conclusion, we demonstrated that intravenously injected cMSCs are capable of suppressing AD via inhibition of IL-4 and IgE production in $\mathrm{T}$ cells and $\mathrm{B}$ cells, respectively. In addition, cMSCs migrated to draining lymph nodes and the inflamed skin lesion that may result in the inhibition of effector cell migration (Supplementary Figure S5). There was a report of a clinical trial using adipose tissue-derived MSCs for the treatment of four AD patients, showing decreased clinical scores and inflammatory responses. ${ }^{48}$ Our findings along with the human clinical trial data could open the door for the development of a new therapeutic strategy to treat $A D$ patients.

\section{Materials and Methods}

The cMSC culture. The cMSCs were isolated from the bone marrow of $\mathrm{C} 3 \mathrm{H} /$ $\mathrm{HeN}$ and Balb/c mice according to the SCM protocol ${ }^{23,24}$ and maintained using Dulbecco's modified Eagle Medium (DMEM) low glucose, 10\% fetal bovine serum (Gibco, Grand Island, NY, USA), and $1 \times$ antibiotic-antimycotic solution (Gibco) at $37^{\circ} \mathrm{C}$ in $5 \% \mathrm{CO}_{2}$. Cells from passages $12-25$ were used in this study. The cMSCs were characterized by flow cytometry according to the cell surface antigens and the differentiation potential into adipocytes, chondrocytes, and osteocytes. ${ }^{23}$ The following antibodies were purchased from BD Bioscience (San Diego, CA, USA): CD44 (IM7), CD45 (30F11), CD73 (TY/11.8), CD105 (FAB1320F), CD117 (2B8), MHC II (2G9), Sca-1 (D7). ${ }^{23}$ Mycoplasma in cMSCs was routinely examined by using a mycoplasma detection kit (e-Myco, iNtRON, Sungnam, Korea). Used cMSCs were mycoplasma-free lines.

T-cell proliferation and cytokine assay. To investigate the effect of the cMSC on T-cell proliferation, $2 \times 10^{5}$ splenocytes from Balb/c mice were stimulated with $1 \mu \mathrm{g} / \mathrm{ml}$ of anti-CD3 and anti-CD28 antibodies (BD Bioscience, San Jose, CA, USA), together with $1 \times 10^{4} \mathrm{cMSCs}$ (or indicated otherwise) in a 96-well plate for 3 days. During the final $12-16 \mathrm{~h}$ culture, $1 \mu \mathrm{Ci} /$ well ${ }^{3} \mathrm{H}$-thymidine was added and T-cell proliferation was determined by thymidine incorporation. To detect cytokines, $1 \times 10^{6}$ splenocytes were stimulated with $1 \mu \mathrm{g} / \mathrm{ml}$ of anti-CD3 and anti-CD28 antibodies together with $5 \times 10^{4} \mathrm{cMSCs}$ in a 24-well plate. For the Transwell experiment, 24-well Transwell plates with a $0.4-\mu \mathrm{m}$ pore membrane (Corning, Oneonta, NY, USA) were used to separate the splenocytes from the cMSCs. After $48 \mathrm{~h}$ of stimulation, IFN- $\gamma$ and IL-4 levels were measured by ELISA according to the manufacturer's protocol (BD Bioscience, San Diego, CA, USA).

B-cell proliferation and antibody assay. Naive B cells were purified by EasyStep magnetic beads (STEMCELL Technologies, Vancouver, BC, Canada). Purified cells $\left(2 \times 10^{5}\right.$ cells) were stimulated with either $10 \mu \mathrm{g} / \mathrm{ml}$ LPS or $5 \mu \mathrm{g} / \mathrm{ml}$ anti-CD40 in the presence of $30 \mathrm{ng} / \mathrm{ml}$ recombinant IL-4 (Creagene, Seoul, Korea) and $1 \times 10^{4} \mathrm{cMSC}$ (or indicated otherwise) in a 96-well plate. After 3 days of culture, B-cell proliferation was determined by the incorporation of ${ }^{3} \mathrm{H}$-thymidine $(1 \mu \mathrm{Ci} /$ well). Using flow cytometry, B-cell division was monitored by CFSE (SigmaAldrich, St. Louis, MO, USA) after 3 days of culture. To detect antibody production, $1 \times 10^{6}$ purified B cells were stimulated with LPS/IL-4 or anti-CD40/IL-4 together with $5 \times 10^{4} \mathrm{cMSC}$ in a 24-well plate. For the Transwell experiment, 24-well Transwell plates with a $0.4-\mu \mathrm{m}$ pore membrane (Corning) were used to separate $B$ cells from the cMSCs. After 5 days, IgE and IgG1 were measured by ELISA according to the manufacturer's protocol (BD Bioscience, San Jose, CA, USA). To measure lgE-producing $B$ cells, cells were cultured for 3 days, brefeldin $\mathrm{A}$ was added during the final $6 \mathrm{~h}$ culture period, and then intracellular $\lg \mathrm{E}$ production was detected by flow cytometry.

Nitrite assay. NO production was measured in supernatants collected after $48 \mathrm{~h}$ of culture. Briefly, $50 \mu \mathrm{l}$ of Griess reagent (prepared with reagents from Sigma, St. Louis, MO, USA) was added to $50 \mu \mathrm{l}$ of culture media, and the absorbance was read at $550 \mathrm{~nm}$ using an automated plate reader. Nitrite concentration was calculated from a $\mathrm{NaNO}_{2}$ standard curve.
AD induction. Balb/c mice were used for the experiments. Animal care and all experimental procedures were conducted in accordance with the Guide for Animal Experiments published by the Korea Academy of Medical Science. AD was induced as previously described with some modifications. ${ }^{49}$ We used six mice per group. Briefly, mice were immunized with $10 \mu \mathrm{g}$ of chicken OVA (grade V; Sigma) mixed with $4 \mathrm{mg}$ of aluminum hydroxide (ImjectAlum; Pierce, Rockford, IL, USA) in a volume of $200 \mu \mathrm{l} 3$ times at 1-week intervals (i.e., days 0,7 , and 14). The mice were epicutaneously sensitized with OVA patches on day 21 . OVA $(10 \mu \mathrm{g})$ prepared in $100 \mu \mathrm{l}$ of PBS was contained in a $1 \times 1-\mathrm{cm}$ patch of sterile gauze that was placed on the shaved backs of mice and secured to the skin with a transparent dressing (Tegaderm; 3M, St. Paul, MN, USA). The patch was changed daily, and the skin was kept in contact with the OVA for 7 days. Thereafter, cMSCs $\left(2 \times 10^{5}\right.$ cells) were intravenously injected into mice on days 29,31 , and 33 . The skin was re-exposed to OVA for 7 days. On day 43, sera and skin biopsy specimens were obtained for ELISA and histological examination, respectively. Mast cells, T cells, and eosinophils in skins were determined by toluidine blue, anti-CD3 antibodies, and H\&E staining, respectively. Cells were counted from three randomly selected fields per slide using a microscope. Two slides per mouse were examined. The three fields were first identified by scanning the entire slide at $\times 100$ magnification. The positively stained cells were counted in high-powered-field (HPF, $\times 400)$. AD experiments have been done twice using syngeneic cMSCs and three times using allogeneic cMSCs. Similar results were obtained.

AD severity scoring. The severity of dermatitis was assessed macroscopically according to the following scoring procedure and was expressed as the sum of the individual score grades from 0 (no symptoms), 1 (mild), 2 (moderate), to 3 (severe) for each of the following five signs and symptoms: (1) pruritus/itching, (2) erythema/hemorrhage, (3) edema, (4) excoriation/erosion, and (5) scaling/ dryness, as described previously. ${ }^{50}$

CM-Dil labeling. For detection of cMSCs in mice, cMSCs were labeled with CellTracker CM-Dil (Invitrogen, Carlsbad, CA, USA). Twenty million cells were resuspended in DPBS along with $1 \mu \mathrm{g} / \mathrm{ml}$ of CM-Dil dye, incubated for $5 \mathrm{~min}$ at $37^{\circ} \mathrm{C}$, and further stained at $4^{\circ} \mathrm{C}$ for $15 \mathrm{~min}$. Cells were washed twice with DPBS and resuspended in $1 \mathrm{ml}$ of DPBS for transplantation.

In vivo injection and tracking of cMSCs. For detection experiments of CM-Dil-labeled cMSCs, mice were immunized with $10 \mu \mathrm{g}$ of chicken OVA/Alum for three times at 1-week intervals. The mice were epicutaneously sensitized with OVA patches on day 21. To distinguish lesion and nonlesion, the patch was placed on the left side of mice to compare with the nonpatch side of skin. We used three mice per group. After 1 week, $1 \times 10^{6} \mathrm{CM}$-Dil-labeled cMSCs were delivered by tail vein injection. Mice were killed at 3 days after MSC injection, and various organs (lung, skin, and lymph nodes) were isolated. Each organ was embedded in OCT compound, quickly frozen in liquid nitrogen, and stored at $-80^{\circ} \mathrm{C}$. Sections were cut into 7- $\mu \mathrm{m}$ slices. The labeled MSCs were visualized using a confocal microscope (Fluoview FV1000, Olympus, Tokyo, Japan). CM-Dil-labeled cMSCs were counted from three randomly selected fields per slide using a microscopy. Two slides per mouse were examined. The three fields were first identified by scanning the entire slide at $\times 100$ magnification. The positively stained cells were quantified by counting cells in HPF $(\times 400)$. Similar results were obtained in two independent experiments

Isolation of RNA and RT-PCR analysis. Total RNA was extracted from $B$ cells using easy-BLUE ( $\mathrm{iNtRON}$ ) and cDNA was prepared using a QuantiTect Reverse Transcription Kit (Qiagen, Hilden, Germany) according to the manufacturer's instructions. The CDNA products were amplified with SYBR Green Quantitative PCR Master Mix (Applied Biosystems, Foster City, CA, USA). The primers (QuantiTect Primer Assays) are all commercially available and validated by Qiagen. Reactions were carried out in the StepOnePlus RealTime PCR System (Applied Biosystems); the conditions for PCR running were as follows: for activating the DNA polymerase, hot start was performed for $10 \mathrm{~min}$ at $95^{\circ} \mathrm{C}$, then cycling at $95^{\circ} \mathrm{C}$ for $15 \mathrm{~s}$ and $60^{\circ} \mathrm{C}$ for $1 \mathrm{~min}$ for a total of 40 cycles.

PCR for $\varepsilon$-GLT, $\varepsilon$-PST, and GAPDH was performed using standard procedures. Briefly, PCR was performed in a total volume of $20 \mu \mathrm{l}$ as follows: (1) denaturation: 5 min at $94^{\circ} \mathrm{C}$; (2) amplification: 35 cycles for $\varepsilon$-GLT and $\varepsilon$-PST, and 25 cycles for GAPDH, $30 \mathrm{~s}$ at $94^{\circ} \mathrm{C}, 30 \mathrm{~s}$ at $58^{\circ} \mathrm{C}$, and $30 \mathrm{~s}$ at $72^{\circ} \mathrm{C}$; and (3) extension: 
$10 \mathrm{~min}$ at $72^{\circ} \mathrm{C}$. Then, $10 \mu \mathrm{l}$ of each sample was run on a $1 \%$ agarose gel, stained with ethidium bromide, and viewed using ultraviolet light. PCR primers used were: $\varepsilon$-GLT: sense primer $5^{\prime}$-GCACAGGGGGCAGAAGAT- $3^{\prime}$ and antisense $5^{\prime}$-CGT TGAATGATGGAGGAT-3'; $\varepsilon$-PST: sense primer $5^{\prime}$-CCAGGGTCATGGAAGC AGTG- $3^{\prime}$ and antisense $5^{\prime}$-CTCTGGCCCTGCTTATTGTTG- $3^{\prime}$ and GAPDH: sense primer $5^{\prime}$-CATCTTCCAGGAGCCAGACC- $3^{\prime}$ and antisense $5^{\prime}$-TCCACCACC CTGTTGCTGTA-3'.

Immunoblotting. B-cell lysates were prepared for sodium dodecyl sulfatepolyacrylamide gel electrophoresis (SDS-PAGE), followed by immunoblotting as previously described. ${ }^{51}$ Antibodies for AID, BLIMP-1, and $\beta$-Actin were purchased from Santa Cruz Biotechnology (Dallas, TX, USA).

Statistical analysis. Student's $t$-test was used to compare two independent groups in which the data were normally distributed. The statistical significances are indicated in the figure legend.

\section{Conflict of Interest}

The authors declare no conflict of interest.

Acknowledgements. We thank all people in the laboratory for their support in this study. This study was supported by Basic Science Research Program and the Bio \& Medical Technology Development Program through the National Research Foundation of Korea (NRF) funded by the Ministry of Science, ICT and Future Planning (NRF-2011-0013452, NRF-2011-0019634, and NRF-2011-0019637), Inha University Research Grant (INHA-47336-01), and Inha hospital Grant.

1. Deckers IA, McLean S, Linssen S, Mommers $M$, van Schayck $C P$, Sheikh $A$. Investigating international time trends in the incidence and prevalence of atopic eczema 1990-2010: a systematic review of epidemiological studies. PLoS One 2012; 7: e39803.

2. Hamid Q, Boguniewicz M, Leung DY. Differential in situ cytokine gene expression in acute versus chronic atopic dermatitis. J Clin Invest 1994; 94: 870-876.

3. Misery L. Therapeutic perspectives in atopic dermatitis. Clin Rev Allergy Immunol 2010; 41 : 267-271.

4. Berke R, Singh A, Guralnick M. Atopic dermatitis: an overview. Am Fam Physician 2012; 86: 35-42.

5. Singer NG, Caplan Al. Mesenchymal stem cells: mechanisms of inflammation. Annu Rev Pathol 2011; 6: 457-478.

6. Uccelli A, Moretta L, Pistoia V. Mesenchymal stem cells in health and disease. Nat Rev Immunol 2008; 8: 726-736.

7. Le Blanc K, Rasmusson I, Sundberg B, Gotherstrom C, Hassan M, Uzunel M et al. Treatment of severe acute graft-versus-host disease with third party haploidentical mesenchymal stem cells. Lancet 2004; 363: 1439-1441.

8. Augello A, Tasso R, Negrini SM, Cancedda R, Pennesi G. Cell therapy using allogeneic bone marrow mesenchymal stem cells prevents tissue damage in collagen-induced arthritis. Arthritis Rheum 2007; 56: 1175-1186.

9. Gonzalez-Rey E, Gonzalez MA, Varela N, O'Valle F, Hernandez-Cortes P, Rico L et al. Human adipose-derived mesenchymal stem cells reduce inflammatory and $T$ cell responses and induce regulatory $T$ cells in vitro in rheumatoid arthritis. Ann Rheum Dis 2010; 69: 241-248.

10. Zappia E, Casazza S, Pedemonte E, Benvenuto F, Bonanni I, Gerdoni E et al. Mesenchymal stem cells ameliorate experimental autoimmune encephalomyelitis inducing T-cell anergy. Blood 2005; 106: 1755-1761.

11. Sun L, Wang $D$, Liang J, Zhang $H$, Feng $X$, Wang $H$ et al. Umbilical cord mesenchymal stem cell transplantation in severe and refractory systemic lupus erythematosus. Arthritis Rheum 2010; 62: 2467-2475.

12. Nemeth K, Leelahavanichkul A, Yuen PS, Mayer B, Parmelee A, Doi K et al. Bone marrow stromal cells attenuate sepsis via prostaglandin $\mathrm{E}(2)$-dependent reprogramming of host macrophages to increase their interleukin-10 production. Nat Med 2009; 15: 42-49.

13. Jung KH, Song SU, Yi T, Jeon MS, Hong SW, Zheng HM et al. Human bone marrowderived clonal mesenchymal stem cells inhibit inflammation and reduce acute pancreatitis in rats. Gastroenterology 2011; 140: 998-1008.

14. Gonzalez-Rey E, Anderson P, Gonzalez MA, Rico L, Buscher D, Delgado M. Human adult stem cells derived from adipose tissue protect against experimental colitis and sepsis. Gut 2009; 58: 929-939.

15. Karussis D, Kassis I. The potential use of stem cells in multiple sclerosis: an overview of the preclinical experience. Clin Neurol Neurosurg 2008; 110: 889-896.
16. Krampera M, Cosmi L, Angeli R, Pasini A, Liotta F, Andreini A et al. Role for interferongamma in the immunomodulatory activity of human bone marrow mesenchymal stem cells. Stem Cells 2006; 24: 386-398.

17. Chen L, Tredget EE, Wu PY, Wu Y. Paracrine factors of mesenchymal stem cells recruit macrophages and endothelial lineage cells and enhance wound healing. PLoS One 2008; 3: e1886.

18. Polchert D, Sobinsky J, Douglas G, Kidd M, Moadsiri A, Reina E et al. IFN-gamma activation of mesenchymal stem cells for treatment and prevention of graft versus host disease. Eur J Immunol 2008; 38: 1745-1755.

19. Cho KS, Park HK, Park HY, Jung JS, Jeon SG, Kim YK et al. IFATS collection: immunomodulatory effects of adipose tissue-derived stem cells in an allergic rhinitis mouse model. Stem Cells 2009; 27: 259-265.

20. Kavanagh $\mathrm{H}$, Mahon BP. Allogeneic mesenchymal stem cells prevent allergic airway inflammation by inducing murine regulatory T cells. Allergy 2011; 66: 523-531.

21. Nemeth K, Keane-Myers A, Brown JM, Metcalfe DD, Gorham JD, Bundoc VG et al. Bone marrow stromal cells use TGF-beta to suppress allergic responses in a mouse model of ragweed-induced asthma. Proc Natl Acad Sci USA 2010; 107: 5652-5657.

22. Bonfield TL, Nolan Koloze MT, Lennon DP, Caplan Al. Defining human mesenchymal stem cell efficacy in vivo. J Inflamm (Lond) 2010; 7: 51.

23. Jeon MS, Yi TG, Lim HJ, Moon SH, Lee MH, Kang JS et al. Characterization of mouse clonal mesenchymal stem cell lines established by subfractionation culturing method. World J Stem Cells 2011; 3: 70-82.

24. Song SU, Kim CS, Yoon SP, Kim SK, Lee MH, Kang JS et al. Variations of clonal marrow stem cell lines established from human bone marrow in surface epitopes, differentiation potential, gene expression, and cytokine secretion. Stem Cells Dev 2008; 17: $451-461$

25. Asari S, Itakura S, Ferreri K, Liu CP, Kuroda Y, Kandeel F et al. Mesenchymal stem cells suppress B-cell terminal differentiation. Exp Hematol 2009; 37: 604-615.

26. Moon HB, Severinson E, Heusser C, Johansson SG, Moller G, Persson U. Regulation of $\operatorname{IgG1}$ and $\operatorname{lgE}$ synthesis by interleukin 4 in mouse B cells. Scand J Immunol 1989; 30: 355-361.

27. Jabara HH, Fu SM, Geha RS, Vercelli D. CD40 and IgE: synergism between anti-CD40 monoclonal antibody and interleukin 4 in the induction of $\operatorname{lgE}$ synthesis by highly purified human B cells. J Exp Med 1990; 172: 1861-1864.

28. Bacharier LB, Geha RS. Molecular mechanisms of IgE regulation. J Allergy Clin Immunol 2000; 105(2 Pt 2): S547-S558.

29. Geha RS, Jabara HH, Brodeur SR. The regulation of immunoglobulin E class-switch recombination. Nat Rev Immunol 2003; 3: 721-732.

30. Shapiro-Shelef M, Calame K. Regulation of plasma-cell development. Nat Rev Immunol 2005; 5: 230-242.

31. Ryan JM, Barry FP, Murphy JM, Mahon BP. Mesenchymal stem cells avoid allogeneic rejection. J Inflamm (Lond) 2005; $2: 8$.

32. Kootiratrakarn T, Fujimura T, Sano K, Okuyama R, Aiba S, Tagami H et al. Development of a novel Ag-specific immunotherapy using $\mathrm{CpG}$ oligodeoxynucleotides in a new, unique mouse cutaneous eosinophilic inflammation model. Eur J Immunol 2005; 35: 3277-3286.

33. Lu X, Liu T, Gu L, Huang C, Zhu H, Meng W et al. Immunomodulatory effects of mesenchymal stem cells involved in favoring type 2 T cell subsets. Transpl Immunol 2009; 22: $55-61$.

34. Batten P, Sarathchandra P, Antoniw JW, Tay SS, Lowdell MW, Taylor PM et al. Human mesenchymal stem cells induce T cell anergy and downregulate T cell allo-responses via the $\mathrm{TH} 2$ pathway: relevance to tissue engineering human heart valves. Tissue Eng 2006; 12: 2263-2273.

35. Bai L, Lennon DP, Eaton V, Maier K, Caplan Al, Miller SD et al. Human bone marrowderived mesenchymal stem cells induce Th2-polarized immune response and promote endogenous repair in animal models of multiple sclerosis. Glia 2009; 57: 1192-1203.

36. Aggarwal S, Pittenger MF. Human mesenchymal stem cells modulate allogeneic immune cell responses. Blood 2005; 105: 1815-1822.

37. Deng W, Han Q, Liao L, You S, Deng H, Zhao RC. Effects of allogeneic bone marrowderived mesenchymal stem cells on T and B lymphocytes from BXSB mice. DNA Cell Biol 2005; 24: 458-463.

38. Beyth S, Borovsky Z, Mevorach D, Liebergall M, Gazit Z, Aslan H et al. Human mesenchymal stem cells alter antigen-presenting cell maturation and induce T-cell unresponsiveness. Blood 2005; 105: 2214-2219.

39. Comoli P, Ginevri F, Maccario R, Avanzini MA, Marconi M, Groff A et al. Human mesenchymal stem cells inhibit antibody production induced in vitro by allostimulation. Nephrol Dial Transplant 2008; 23: 1196-1202.

40. Yoo HS, Yi T, Cho YK, Kim WC, Song SU, Jeon MS et al. Isolated by different isolation methods show variations in the regulation of graft-versus-host disease. Immune Network 2013; 13: 133-140.

41. Klein U, Dalla-Favera R. Germinal centres: role in B-cell physiology and malignancy. Nat Rev Immunol 2008; 8: 22-33.

42. Zan H, Casali P. Regulation of Aicda expression and AID activity. Autoimmunity 2013; 46: 83-101.

43. Sun SC. Non-canonical NF-kappaB signaling pathway. Cell Res 2011; 21: 71-85.

44. Teng G, Hakimpour P, Landgraf P, Rice A, Tuschl T, Casellas R et al. MicroRNA-155 is a negative regulator of activation-induced cytidine deaminase. Immunity 2008; 28 . $621-629$. 
45. Basso K, Schneider C, Shen Q, Holmes AB, Setty M, Leslie C et al. BCL6 positively regulates AID and germinal center gene expression via repression of miR-155. J Exp Med 2012; 209: 2455-2465

46. Silva Jr WA, Covas DT, Panepucci RA, Proto-Siqueira R, Siufi JL, Zanette DL et al. The profile of gene expression of human marrow mesenchymal stem cells. Stem Cells 2003; 21: $661-669$

47. Yoshimoto T, Okada K, Morishima N, Kamiya S, Owaki T, Asakawa M et al. Induction of IgG2a class switching in B cells by IL-27. J Immunol 2004; 173: 2479-2485.

48. Ra JC, Kang SK, Shin IS, Park HG, Joo SA, Kim JG et al. Stem cell treatment for patients with autoimmune disease by systemic infusion of culture-expanded autologous adipose tissue derived mesenchymal stem cells. J Transl Med 2011; 9: 181.

49. Jin H, He R, Oyoshi M, Geha RS. Animal models of atopic dermatitis. J Invest Dermatol 2009; 129: 31-40

50. Taniguchi Y, Kohno K, Inoue S, Koya-Miyata S, Okamoto I, Arai N et al. Oral administration of royal jelly inhibits the development of atopic dermatitis-like skin lesions in $\mathrm{NC} / \mathrm{Nga}$ mice. Int Immunopharmacol 2003; 3: 1313-1324.
51. Jeon MS, Atfield A, Venuprasad K, Krawczyk C, Sarao R, Elly C et al. Essential role of the E3 ubiquitin ligase Cbl-b in T cell anergy induction. Immunity 2004; 21: 167-177.

Cell Death and Disease is an open-access journal

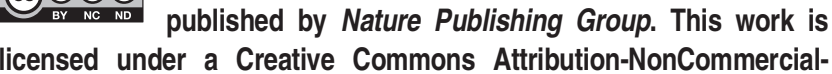
NoDerivs 3.0 Unported License. The images or other third party material in this article are included in the article's Creative Commons license, unless indicated otherwise in the credit line; if the material is not included under the Creative Commons license, users will need to obtain permission from the license holder to reproduce the material. To view a copy of this license, visit http://creativecommons.org/licenses/ by-nc-nd/3.0/

Supplementary Information accompanies this paper on Cell Death and Disease website (http://www.nature.com/cddis) 